\title{
Erratum to: Quantitative 18F-fluorocholine positron emission tomography for prostate cancer: correlation between kinetic parameters and Gleason scoring
}

Joshua D. Schaefferkoetter ${ }^{1,2^{*}}$, Ziting Wang ${ }^{3}$, Mary C. Stephenson ${ }^{1}$, Sharmili Roy ${ }^{1}$, Maurizio Conti ${ }^{4}$, Lars Eriksson ${ }^{4}$, David W. Townsend ${ }^{1,2}$, Thomas Thamboo ${ }^{5}$ and Edmund Chiong ${ }^{3}$

\section{Erratum}

Upon publication of the original article [1], it was noticed that the author "Joshua D. Schaefferkoetter" was incorrectly included as "Joshua J.D. Schaefferkoetter".

\section{Author details}

'A*STAR-NUS Clinical Imaging Research Centre, Centre for Translational, Medicine (MD6), 14 Medical Drive, \#B1-01, Singapore 117599, Singapore. ${ }^{2}$ Department of Diagnostic Radiology, National University Hospital, Singapore, Singapore. ${ }^{3}$ Department of Urology, National University Health, System, Singapore, Singapore. ${ }^{4}$ Siemens Healthcare Molecular Imaging, Knoxville, TN 37919, USA. ${ }^{5}$ Department of Pathology, National University,

Health System, Singapore, Singapore.

Received: 2 June 2017 Accepted: 2 June 2017

Published online: 21 June 2017

\section{Reference}

1. Schaefferkoetter JD, et al. Quantitative ${ }^{18} \mathrm{~F}$-fluorocholine positron emission tomography for prostate cancer: correlation between kinetic parameters and Gleason scoring. EJNMMI Research. 2017;7:25. doi:10.1186/s13550-017-0269.

\footnotetext{
*Correspondence: dnrjds@nus.edu.sg

'A*STAR-NUS Clinical Imaging Research Centre, Centre for Translational,

Medicine (MD6), 14 Medical Drive, \#B1-01, Singapore 117599, Singapore

${ }^{2}$ Department of Diagnostic Radiology, National University Hospital,

Singapore, Singapore

Full list of author information is available at the end of the article
} 